\title{
Communicable Diseases Report, NSW, January-March 2013
}

\section{Communicable Diseases Branch \\ Health Protection NSW}

For updated information, including data and facts on specific diseases, visit www.health.nsw.gov.au and click on Public Health and then Infectious Diseases. The communicable diseases site is available at: http://www.health.nsw.gov.au/publichealth/ infectious/index.asp.

Figure 1 and Table 2 show notifications of communicable diseases with onset from January to March 2013 in New South Wales (NSW).

\section{Enteric infections}

\section{Outbreaks of suspected foodborne disease}

There were 12 outbreaks of foodborne or suspected foodborne disease reported by members of the public or identified through routine surveillance of Salmonella data in the first quarter of 2013. Five outbreaks were due to Salmonella Typhimurium, one each was due to norovirus, Salmonella Birkenhead, and Salmonella Cerro, and the others were due to unknown pathogens.

Only two investigations were able to provide sufficient evidence to identify the source of the infection. In one of these a Public Health Unit investigated three groups of people that ate at a restaurant in February 2013 and subsequently became ill with gastrointestinal illness. Seven out of a total 10 people consumed fried ice cream and all of these developed illness. All cases had stool samples that tested positive for Salmonella Typhimurium (MLVA 3-9-7-14-523 or 3-9-8-14-523). The NSW Food Authority (NSWFA) inspected the restaurant and took samples of frozen and cooked fried ice cream; these samples also tested positive for Salmonella Typhimurium (MLVA 3-9-7-14-523 or 3-9-8-14-523). The restaurant proprietor was warned about the risks of preparing fried ice cream with raw eggs and was fined for the sale of unsafe food. The NSWFA also inspected the egg farm that supplied the restaurant and found Salmonella with the same MLVA pattern on an egg rinse sample. In the other outbreak a cluster of Salmonella Typhimurium (MLVA 3-17-9-12-523) cases were notified by a hospital from a family of four. They were admitted to a hospital with salmonellosis in March 2013. The only common risk food consumed prior to this was banana smoothies made with milk and raw eggs; they had been eating these smoothies daily. The eggs came from a small boutique free-range egg farm. The family were provided with information about salmonellosis and the risks involved with eating raw eggs, and have since discontinued this practice.

\section{Viral gastrointestinal disease}

There were 136 reported outbreaks of (suspected) viral gastrointestinal disease in institutions in the first quarter of 2013. Of these, 49 (36\%) occurred in aged-care facilities, $71(52 \%)$ occurred in child-care centres, $14(10 \%)$ in hospitals, and one each in a school and a military institution. The outbreaks affected a total of 1925 people.

In $45 \%(n=61)$ of all outbreaks, one or more stool specimens were laboratory tested to identify a possible cause of the outbreak. Norovirus was identified in 34\% $(n=21)$ of these outbreaks. In seven outbreaks, another pathogen was detected alongside norovirus (rotavirus in one outbreak, Clostridium difficile in three outbreaks, giardia in one outbreak and Salmonella in two outbreaks). Of the 61 outbreaks where one or more stool specimens were tested, $61 \%(n=37)$ of all results were negative for any pathogens.

\section{Respiratory infections Influenza}

Influenza continued to circulate at low levels in the first quarter of 2013, with evidence of co-circulation of influenza $\mathrm{A}(\mathrm{H} 1 \mathrm{~N} 1) 2009$, influenza $\mathrm{A}(\mathrm{H} 3 \mathrm{~N} 2)$, and influenza $\mathrm{B}$ strains. The number of influenza cases in January and February was slightly higher than the historical average for this time of year.

Influenza activity was also measured by the number of people who presented to 59 selected NSW emergency departments with influenza-like-illness (ILI). Presentations for ILI were also at low levels but slightly above seasonal averages in January and February.

For a more detailed report on respiratory activity in NSW see: http://www.health.nsw.gov.au/PublicHealth/Infectious/ influenza_reports.asp. 


\section{Legionellosis}

There were 13 cases of legionellosis due to Legionella pneumophila strains notified in the first quarter of 2013, a marked decrease from the 25 notified for the same period in 2012. There were also six notifications of legionellosis due to L. longbeachae strains, similar to the previous year (seven cases).

\section{Vaccine-preventable diseases Meningococcal disease}

Eight cases of meningococcal disease were notified in NSW in the first quarter of 2013 (four in January and four in March), an increase from six notified for the same period in 2012. The age of the cases ranged from 10 months to 67 years, with five cases aged less than 5 years. Of the eight notifications, six $(75 \%)$ were due to serogroup B (for which there is no vaccine), and two (25\%) were due to serogroup $\mathrm{C}$. The two cases of meningococcal disease caused by serogroup $\mathrm{C}$ were reported in adult women, one of whom (a woman aged in her fifties) died.

Immunisation against meningococcal $\mathrm{C}$ disease is recommended for all children at the age of 12 months, as well as people at high risk of disease.

\section{Measles}

One case of measles was notified in NSW in the first quarter of 2013 (February). The case was an infant from South Western Sydney Local Health District, who acquired her infection in Pakistan. Public health measures were implemented and no further transmission was identified in association with this case.

Two doses of measles-containing vaccine are recommended for all children at 12 and 18 months age. All young adults planning international travel should ensure they have had two doses of measles-containing vaccine in their lifetime before they travel.

\section{Pertussis}

There were 637 pertussis cases notified in NSW during the first quarter of 2013 (293 in January, 202 in February and 142 in March). This is approximately one-third of the 2064 notifications for the same period in 2012, and represents the lowest number of notifications for a first quarter since 2007. Most cases were in the 0-4-year age group $(n=155)$, followed by the 5-9 $(n=133)$ and $10-14$-year age groups $(n=56)$.

Direct protection for young infants remains available through free vaccination for pertussis that is administered at 2, 4 and 6 months of age. The first dose can be provided as early as 6 weeks of age, with a booster dose at $3 \frac{1}{2}$ to 4 years. Whooping cough vaccination is strongly recommended for adults in contact with young babies too young to be vaccinated. Women planning a pregnancy or in their third trimester are encouraged to receive a whooping cough vaccine on prescription to protect their very young babies.

\section{Sexually transmissible infections and bloodborne viruses \\ Chlamydia}

There were 5338 cases of chlamydia notified in NSW during the first quarter of 2013. This number is slightly above the 5-year average of 5000 notifications for this quarter in previous years.

\section{Gonorrhoea}

There were 1097 cases of gonorrhoea notified in NSW during the first quarter of 2013, a $12 \%$ increase compared with the same period in $2012(n=979)$. Over the past 5 years the majority of notifications $(80 \%)$ have been reported in males, with the highest number $(n=935)$ in the 25-29-year age group accounting for $22 \%$ of all notifications.

\section{Syphilis}

There were 239 cases of syphilis reported in NSW in the first quarter of 2013, an increase of $24 \%$ compared with the same period in 2012. Of the syphilis cases for the first quarter, 152 (64\%) were classified as infectious syphilis.

\section{Lymphogranuloma venereum}

There were nine cases of lymphogranuloma venereum notified during the first quarter of 2013 compared to three notified during the first quarter of 2012. All cases have occurred in males, ranging in age from 18 to 75 years.

HIV

There were 78 cases of newly diagnosed HIV infection notified in NSW residents during the first quarter of 2013, a decrease from 112 notifications in same period in 2012 and from the first quarter 4-year average of 93. Similar to previous years, $77 \%$ of infections were reported to be homosexually acquired and 13\% were heterosexually acquired (in people not from high HIV prevalence countries). The highest number of notifications was amongst people aged 20-29 years.

More than one-third (36\%) of the notifications were reported as recent HIV infection at time of diagnosis (defined as either a negative or indeterminate HIV antibody test or seroconversion illness in the previous 12 months), while 15\% were advanced infections (AIDS and/or CD 4200 cells $/ \mu \mathrm{L}$ ). Of the 78 people notified, $42 \%$ had commenced treatment soon after diagnosis.

A summary of 2012 notification data for HIV is available at: www.health.nsw.gov.au/Infectious/hiv/Documents/ 2012-hiv-summary.pdf. 


\section{Arboviral infections}

A total of 339 cases of arboviral infection were notified in NSW residents during the first quarter of 2013, a reminder of the importance of taking measures to avoid mosquito bites both in Australia and whilst travelling overseas. There were no cases of Murray Valley encephalitis or Kunjin virus infection reported in NSW residents during this period.

\section{Ross River virus}

There were 116 cases of Ross River virus infection notified in the first quarter of 2013. This was a substantial decrease from previous years, with 208 cases and 263 cases reported for the same period in 2012 and 2011 respectively. Notifications of Ross River virus infection were generally highest in coastal regions and lower in inland parts of the state during this period, likely reflecting rainfall patterns and mosquito activity.

\section{Barmah Forest virus}

There were 134 cases of Barmah Forest virus infection notified in NSW during this period, an increase over the same period for 2012 in which 105 cases were reported. The figure for 2013 should be interpreted with caution however as Health Protection NSW is investigating concerns of false positive results in this period.

\section{Chikungunya virus}

Four cases of Chikungunya virus infection were notified in NSW in the first quarter of 2013. All cases were acquired overseas in countries where Chikungunya is known to be endemic.

\section{Dengue virus}

There were 54 cases of dengue virus infection notified in NSW during this period, a decrease from 96 cases reported in the same period in 2012. All cases in the first quarter of 2013 were overseas-acquired infections, excluding one case in which country of acquisition was not recorded. Thailand and Indonesia accounted for almost half of infections, with $23 \%$ of notified cases being acquired in each. Thirteen percent of cases were acquired in the Philippines and $11 \%$ in India.

\section{NSW Denominator Data Project}

Notifications of positive laboratory results for notifiable conditions provide information about the number of new cases of disease. Data on the level of testing is useful to indicate whether an apparent increase in notification may be due to increased testing.

In 2012, NSW Health commenced the NSW Denominator Data Project to collect the total number of tests performed per month (the denominator data) for 10 selected notifiable conditions for which the testing rate might impact the notification rate. Data were requested from 14 public and private laboratories in NSW. The data were collated to give monthly aggregated data per condition. No demographic information was provided.

The positivity rate for all conditions from January 2012 to March 2013 ranged from $0.1 \%$ (shigellosis) to $5.7 \%$ (chlamydia infection) (Table 1). Notifications for chlamydia and gonorrhoea were correlated with testing, while the incidence of enteric conditions suggests that seasonal factors rather than testing patterns influence notification rates.

Table 1. Number and positivity (\%) of tests performed for denominator data collected between January 2012 and March 2013

\begin{tabular}{llcc}
\hline Condition & Tests & Number of tests & Positivity (\%) \\
\hline Chlamydia & C. trachomatis nucleic acid test (NAT) & 468356 & 5.7 \\
Gonorrhoea & N gonorrhoeae NAT, culture & 698907 & 0.7 \\
HIV & Serology & 495661 & Not reported \\
Ross River virus infection & Serology & 22558 & 3.2 \\
Barmah Forest virus infection & Serology & 17270 & 2.8 \\
Pertussis & NAT, serology, culture & 166593 & 3.9 \\
Salmonellosis & NAT, culture & 234056 & 1.7 \\
Shigellosis & & & 0.1 \\
Cryptosporidiosis & Antigen, microscopy & 202065 & 0.6 \\
Giardiasis & & & 1.3 \\
\hline
\end{tabular}


An investigation of Salmonella Typhimurium linked to contaminated eggs on the Central Coast of NSW, 2008

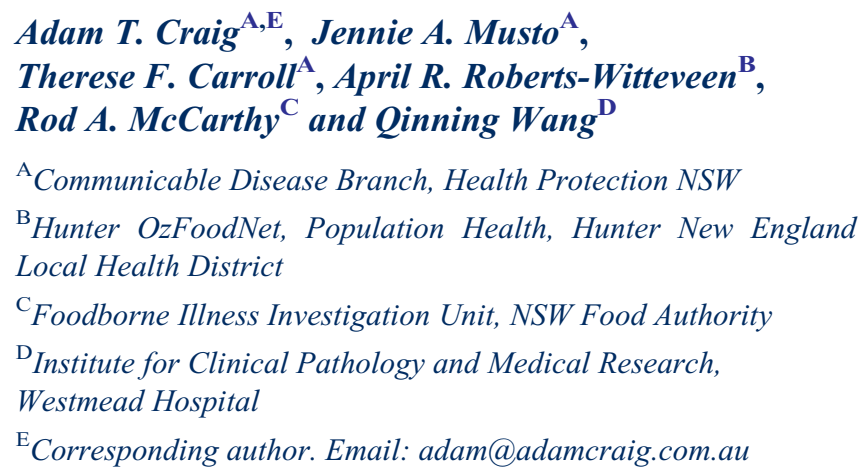

Salmonella is a common cause of foodborne illness in Australia with an estimated 48763 people (257 per 100000 population) infected per year. ${ }^{1}$ It is the most frequently notified enteric disease in New South Wales (NSW), with $S$. Typhimurium the most commonly identified serovar in outbreaks. $^{2}$

In NSW, all Salmonella isolates from public and private laboratories are routinely sent to the NSW Enteric Reference Laboratory, Institute for Clinical Pathology and Medical Research (ICPMR), Westmead Hospital, for serotyping. $S$. Typhimurium isolates are then further subtyped using multilocus variable number tandem repeat analysis (MLVA). ${ }^{3}$ For $S$. Typhimurium, five loci on the gene are used for tandem repeat analysis; the isolates with the same MLVA patterns are considered to be indistinguishable. ${ }^{4}$ ICPMR reports notifiable enteric disease data to the Communicable Diseases Branch of Health Protection NSW daily, where the data are monitored as part of routine surveillance.

In February 2008, epidemiologists from the then NSW Department of Health identified nine $S$. Typhimurium cases with identical MLVA patterns geographically clustered around a town on the Central Coast of NSW. This paper describes the investigation of this point-source outbreak, using MLVA typing, and the public health response.

\section{Methods \\ Epidemiological investigation}

Upon detection of the outbreak a case-series analysis was conducted. A case was defined as a resident of NSW with gastroenteritis and $S$. Typhimurium MLVA 3-17-16-13523 (or a related strain) identified from a stool specimen collected from 14 January 2008 to 4 May 2008. MLVA profiles that varied by one or two repeats at loci 2,3 or 4 were viewed as a 'related strain'.

Active case finding was undertaken through the local hospital and private laboratories, alerting them to the outbreak and ensuring priority transportation of all Salmonella isolates to ICPMR for further characterisation.

Identified cases were interviewed by telephone using a standard hypothesis-generating questionnaire. The questionnaire collected demographic information, details of symptoms experienced, as well as food and other potential exposures in the 7 days prior to illness onset. The information collected from these questionnaires was used to develop a targeted questionnaire that was subsequently used in case interviews.

Data were collated and analysed using Microsoft Excel.

\section{Environmental investigation}

The NSW Food Authority (NSWFA) inspected sites (including a local egg farm), which, as a result of the epidemiological investigation, were suspected of being potential sources of the outbreak. At the farm in question the NSWFA collected eggs and environmental samples from laying sheds for microbial analysis. Environmental samples were tested for Salmonella by ICPMR and were typed using the MLVA method.

The NSWFA traced the farm's supplier of hatchlings and chicken feed, as well as the farm's regular egg deliveries to commercial kitchens in the local area.

\section{Results}

\section{Epidemiological investigation}

Forty-four cases of $S$. Typhimurium MLVA 3-17-16-13523 (later phage typed as $S$. Typhimurium phage type 126 or $S$. Typhimurium phage type 126 var 1 at the Microbiological Diagnostic Unit - Public Health Laboratory, University of Melbourne) were identified. Of these, 21 (48\%) were interviewed. The 23 cases not interviewed were either not contactable or notification of their infections occurred after the source of the outbreak had been identified, control measures put in place and the investigation closed. Of the 44 confirmed cases, the majority (62\%) were female. Cases were aged from 3 to 91 years (median 34 years). Ninety-five percent of cases lived in the Central Coast region of NSW.

The index case was notified to NSW Health through routine laboratory-based surveillance on 22 February 2008. The index case's specimen was collected on 14 January 2008; when interviewed, the case reported an illness onset date of 13 January 2008. The peak in collection of outbreak strain-positive specimens was in the week beginning 11 February 2008 . The last specimen that tested positive for the outbreak strain was collected in the week beginning 5 May 2008, after the investigation was officially closed (Figure 1).

All of the 21 cases interviewed reported experiencing diarrhoea; one-third reported experiencing bloody 


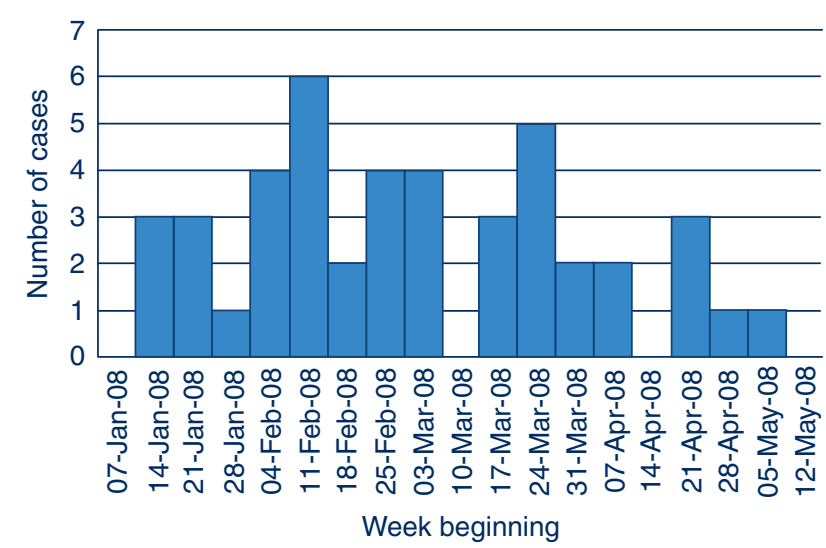

Figure 1. Cases of Salmonella Typhimurium MLVA 3-17-16-13523 notified to NSW Health, January-May 2008 (by specimen collection date).

diarrhoea. Fifty-two percent of cases interviewed reported nausea, $43 \%$ reported vomiting, and $29 \%$ reported fever. Six cases $(29 \%)$ required hospitalisation for their illness. The duration of illness ranged from 4 to 15 days.

Of the 21 cases interviewed, four (19\%) reported purchasing eggs directly from the farm and consuming them prior to onset of illness. A further eight (38\%) ate dishes purchased at local restaurants that, after investigation, were identified as containing eggs sourced from the farm. All other cases reported consuming eggs prior to their illness but could not recall where they purchased them. No other common food sources were identified.

\section{Environmental investigation}

The NSWFA conducted three site visits to the farm over the course of the investigation: on 4 March 2008, 17 March 2008, and 15 April 2008. The farm sold both eggs and chicken manure (for gardening) from a small roadside retail shop and distributed approximately 1800 eggs to local restaurants per week. A list of commercial kitchens to which the farm distributed eggs was obtained, resulting in the identification of six previously unlinked cases (two of which were outside the local area).

Results for the chicken faecal samples collected from two of the five cage lines and from a cloacal swab taken from a dead chicken (in a different cage line from the two where faecal samples were taken) were positive for $S$. Typhimurium MLVA 3-17-16-13-523.

The investigation of the farm's supplier of hatchlings and chicken feed identified that hatchlings were sourced from a major supplier with national distribution and feed was sourced from a large NSW regional supplier. Water used by the farm was sourced from a treated municipal supply. This information was collected as a routine part of the environmental investigation and used to test the hypothesis that the infectious agent may have been introduced to the chicken farm with supplied hatchlings, feed or water. As no cases of salmonellosis with the outbreak strain were identified elsewhere in NSW in the same period the hypothesis was rejected.

\section{Public health intervention}

The local Public Health Unit distributed public information through the media about risk factors for contracting salmonellosis, including reinforcing good food hygiene practices.

The farm was advised to cease 'wet wiping' of faecal matter from the surface of heavily soiled eggs as it is possible this practice increases the spread of Salmonella between eggs.

\section{Discussion}

This paper describes the investigation of a localised, protracted point-source outbreak of $S$. Typhimurium identified through routine surveillance.

Eggs are a known source of Salmonella infections. In 2012, eggs were identified as the cause of $93 \%(13 / 14)$ of foodborne $S$. Typhimurium outbreaks where investigators were able to identify a food vehicle (personal communication, J Musto). The environmental investigation identified Salmonella in three of the five cage lines. This investigation reinforces the need to strengthen Salmonella control measures at the point of production (on-farm control), during retail and wholesale (storage and packaging of eggs and food handling/food safety in food outlets), and at consumer level (food handling and food safety measures).

Washing of eggs is recognised as a risky process that can assist the transfer of Salmonella from the shell of contaminated eggs to the shell of uncontaminated eggs via contaminated washing water or washcloths. ${ }^{5}$ The practice of 'wet wiping' of soiled eggs needs to be addressed through targeted education and tighter regulation.

The use of MLVA typing facilitated the early detection and rapid confirmation of cases, resulting in a faster and more effective public health response, and potentially averting many more cases of illness.

This investigation's limitations include poor food consumption recall by cases interviewed due, in part, to lagtime between sample collection, testing, and interview. Also, 'trace-back' of eggs is difficult as eggs are a commonly-consumed food, often purchased well before they are eaten; as such, recall of place of purchase and brand of eggs is often poor. Tracing the source of eggs sold by retailers or used in restaurants was also problematic, as supply records were often not kept. 


\section{Conclusion}

MVLA typing is a useful tool for the identification of point-source outbreaks of $S$. Typhimurium. The outbreak described in this paper reinforces the need for strengthening Salmonella control at the point of production, retail and consumer preparation to reduce the burden of egg-related salmonellosis in Australia.

\section{Acknowledgments}

We acknowledge the contribution of Craig Shadbolt and Marianne Tegal from the NSW Food Authority; Peter Lewis, Lucy Cook and Richard Broome from the then Northern Sydney Central Coast Area Health Service; and Tory Worgan from Hunter OzFoodNet for their contributions during this investigation.

\section{References}

1. Hall G, Raupach J, Yohannes K. An estimate of under-reporting of foodborne notifiable diseases: Salmonella, Campylobacter and Shiga Toxin producing E. coli (STEC). Working paper no. 52. Canberra: National Centre for Epidemiology and Population Health, Australian National University; 2006.

2. NSW 2011 OzFoodNet Annual Report. Available at: http:// www.health.nsw.gov.au/Infectious/diseases/Documents/ofn_ annual_report_2011.pdf (Cited 18 September 2013).

3. Gilbert GL. Using MLVA to type strains of Salmonella Typhimurium in New South Wales. N S W Public Health Bull 2008; 19(1-2): 29-31. doi:10.1071/NB07116

4. Lindstedt BA, Vardund T, Aas L, Kapperud G. Multiple-locus variable-number tandem-repeats analysis of Salmonella enterica subsp. enterica serovar Typhimurium using PCR multiplexing and multicolor capillary electrophoresis. J Microbiol Methods 2004; 59(2): 163-72. doi:10.1016/j.mimet.2004.06.014

5. Caudill AB, Curtis PA, Anderson KE, Kerth LK, Oyarazabal O, Jones DR et al. The effects of commercial cool water washing of shell eggs on Haugh unit, vitelline membrane strength, aerobic microorganisms, and fungi. Poult Sci 2010; 89(1): 160-8. doi:10.3382/ps.2009-00316 
Figure 1. Reports of selected communicable diseases, NSW, Jan 2005 to Mar 2013, by month of onset.

Preliminary data: case counts in recent months may increase because of reporting delays.

Laboratory-confirmed cases only, except for measles, meningococcal disease and pertussis.

NB: Multiple series in graphs are stacked, except gastroenteritis outbreaks.

NB: Outbreaks are more likely to be reported by nursing homes and hospitals than by other institutions.

NSW Population

Male $\quad 50 \%$

$<5$ y $\quad 7 \%$

$5-24$ y $27 \%$

$25-64$ y $53 \%$

$65+y \quad 13 \%$

Rural $\quad 46 \%$

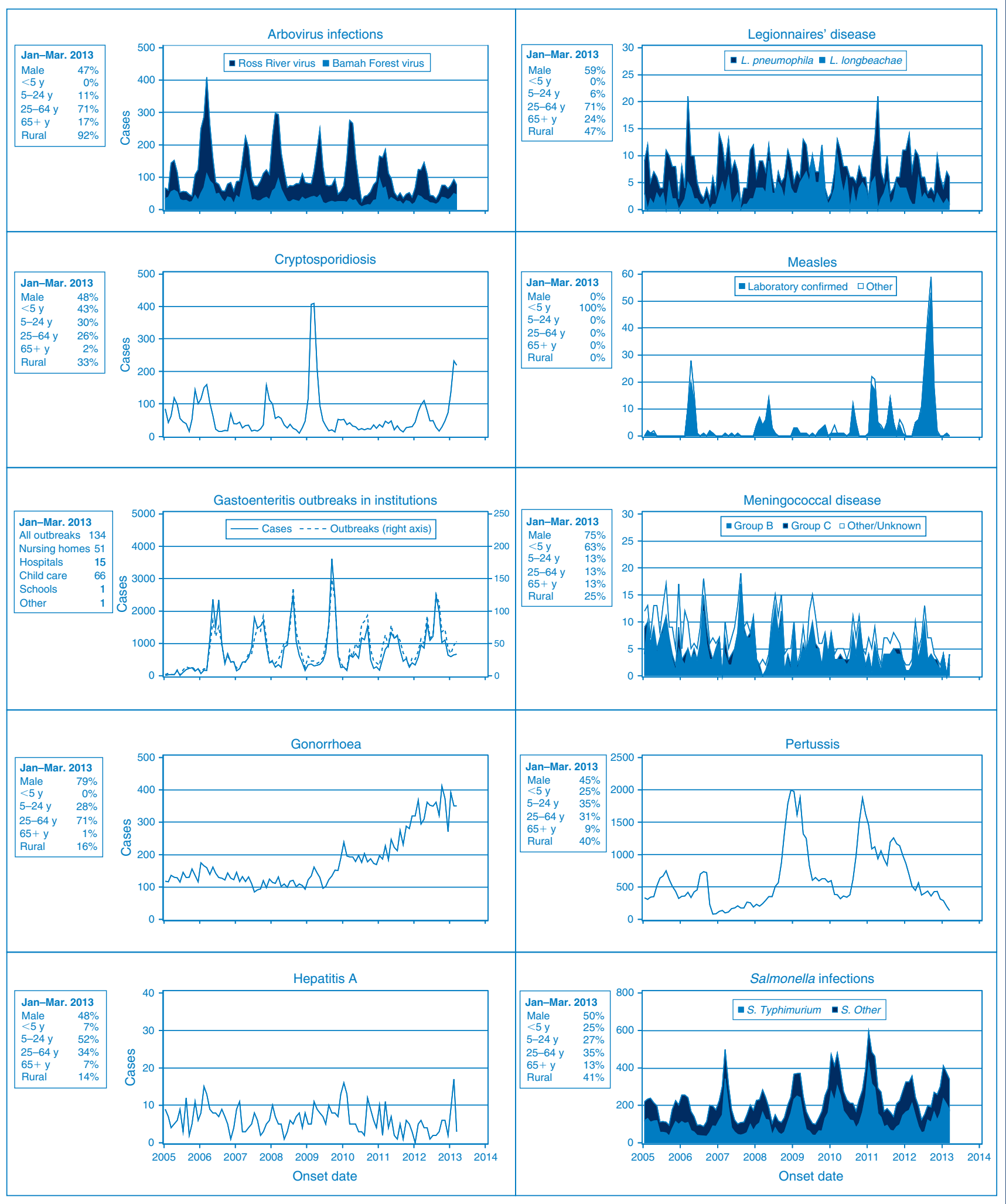




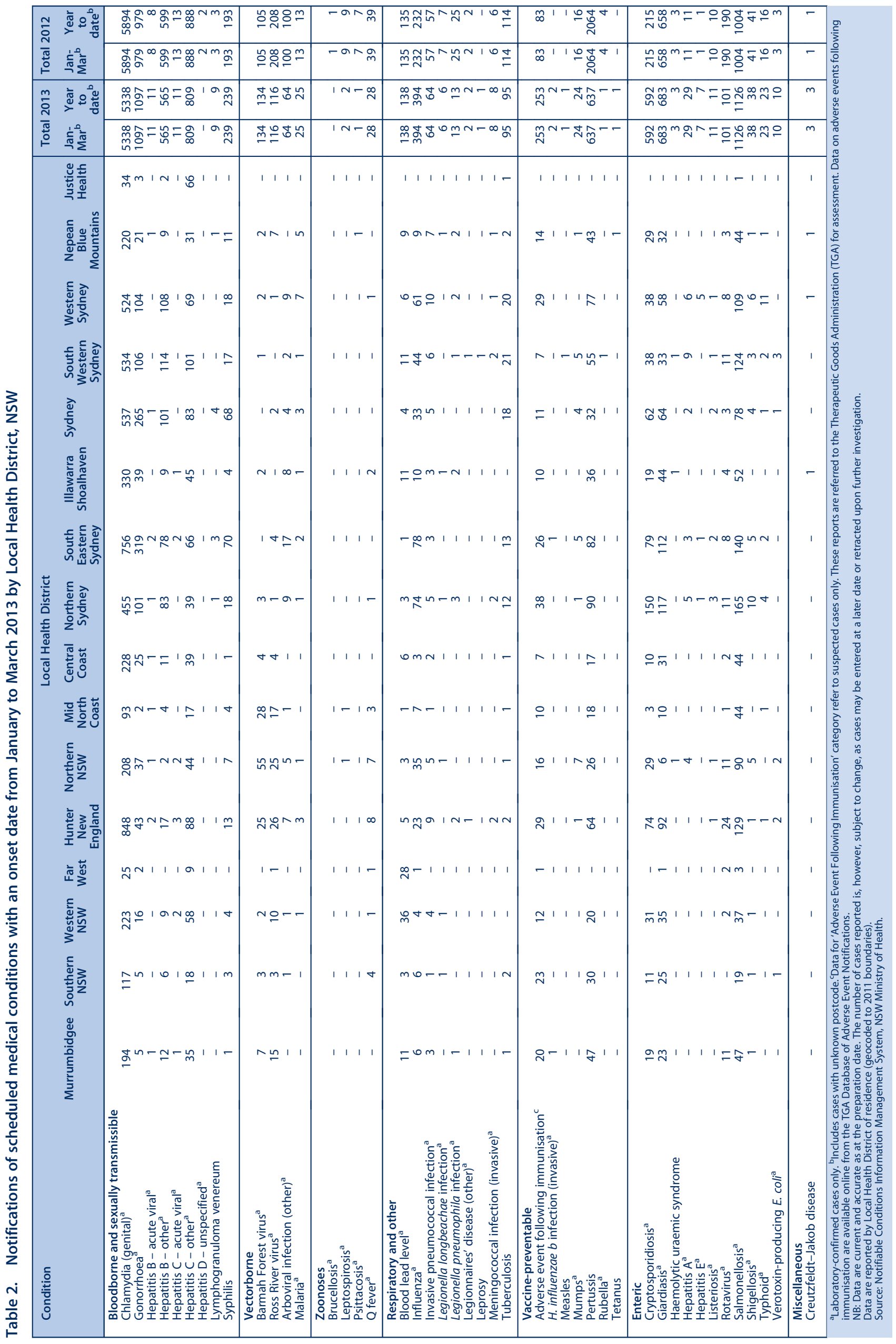

